\title{
LA INFLUENCIA DE LAS VARIEDADES REGIONALES DEL ESPAÑOL EN LA INTERPRETACIÓN SIMULTÁNEA AL CHECO
}

Zuzana BALOUNOVÁ

Universidad Carolina, Praga

\begin{abstract}
En): In many ways, the society in which we live is more globalized and linguistically diverse than ever before. Necessarily, this poses a challenge for interpreters because, in practical terms, it means that Spanish to Czech interpreters are likely to be often in contact with native Spanish speakers not only from Europe but also from Central and South America. Therefore, they should have a profound knowledge of different regional varieties of Spanish. To determinate whether students of Spanish to Czech interpreting are acquainted with these varieties, the author of this article conducted an experiment at the Institute of Translation Studies, Charles University. As part of the experiment, five Master students of conference interpreting were asked to interpret simultaneously a speech pronounced by four different speakers (the speakers were from Argentina, Peru, Mexico and from the North of Spain) and to complete a questionnaire. Overall, the interpreters made fewer omissions, additions, semantic errors and delivery errors while interpreting the part pronounced by the Spanish speaker. Moreover, throughout the speech the interpreters who are in frequent contact (TV, radio) with Latin American varieties of Spanish made fewer omissions, additions, semantic errors and delivery errors than the interpreters who are not in regular contact with these varieties. This suggests that better knowledge of different regional varieties of Spanish may have a positive impact on the quality of simultaneous interpreting.
\end{abstract}

Keywords (En): varieties of Spanish; simultaneous interpreting; interpreting errors; experiment

Palabras clave (Es): variedades del español; interpretación simultánea; errores de interpretación; experimento

\section{Introducción}

En el presente estudio, se expone el experimento llevado a cabo en el Instituto de Traductología de la Facultad de Filosofía y Letras de la Universidad Carolina. Primero, se presentan consideraciones generales sobre las variedades regionales del español en relación con la interpretación simultánea. A continuación, se describe el experimento dedicado al tema de la influencia de las variedades regionales del español en la calidad de la interpretación simultánea al checo y sus resultados. Finalmente, se ofrece una lista de recursos en línea que pueden servir de ayuda a cualquier estudiante de español como lengua extranjera para mejorar su conocimiento de las variedades regionales del español.

\section{Las variedades regionales del español y la interpretación simultánea del español al checo}

Existen numerosas publicaciones que tratan el tema de la calidad de la interpretación simultánea o consecutiva. Sin embargo, la mayoría de estos estudios se centra en la interpretación del inglés y no en la interpretación del español. Las publicaciones examinan, entre otros, el papel del inglés como lengua franca o la influencia del acento no nativo en la calidad de la interpretación. La razón principal 
de esto es que el número de hablantes no nativos del inglés supera al número de hablantes nativos. Obviamente, este no es el caso del español, puesto que al ser la lengua materna de 480 millones de personas, de los cuales más del $90 \%$ proviene de América Latina, el español es la segunda lengua más hablada como lengua nativa, después del chino. ${ }^{1}$ Por este motivo, el presente artículo deja aparte el tema de la diferencia entre los hablantes nativos y no nativos del español y se centra exclusivamente en la diferencia entre las variedades regionales del español.

\subsection{Hispanohablantes en la República Checa}

La decisión de centrarse en las diferentes variedades regionales del español se ve apoyada por los datos de la Oficina de Estadística Checa del final de diciembre de 2018. ${ }^{2}$ A finales de 2018, en la República Checa vivían en total 4625 extranjeros procedentes de los países que tienen el español como lengua oficial: una tercera parte de ellos procedente de España y dos terceras partes de América Central o de América del Sur. ${ }^{3}$ Además, se prevé que los números sigan creciendo. Según los datos de la Oficina de Estadística Checa, entre los años 2016 y 2018 aumentó el número de hispanohablantes que llegaron a la República Checa. Como ejemplo, se puede citar el número de mexicanos, colombianos y venezolanos residentes en la República Checa que, entre los años 2016 y 2018, creció por encima del 29, 36 y el $43 \%$, respectivamente. Dicho de otra forma, los intérpretes con el checo como lengua $\mathrm{A}^{4}$ y el español como lengua $\mathrm{B}$ o $\mathrm{C}$, que desarrollan sus actividades en el mercado checo, se ven obligados a interpretar, en particular, a hablantes de origen latinoamericano, fenómeno que se acentúa en el caso de los intérpretes en los servicios públicos. ${ }^{5}$

\section{2. ¿Por qué es tan necesario que los estudiantes del programa Interpretación checo-español se familiaricen con las variedades regionales del español?}

Varios trabajos dedicados a la teoría y didáctica de la interpretación confirman que un sólido conocimiento de las variedades regionales de la lengua puede mejorar la calidad de la interpretación. A guisa de ejemplo se puede mencionar el artículo «Listening effort and accented speech» de Kristin J. VAN ENGEN y Jonathan E. PEELLE (2014). Según este artículo, que estudia la cuestión de comprensión

\footnotetext{
${ }^{1}$ InSTITUTO CERVANTES (2018), 577 millones de personas hablan español, el 7,6 \% de la población mundial, [cit. 28.8.2019], recuperado de:

https://www.cervantes.es/sobre_instituto_cervantes/prensa/2018/noticias/np_presentacionanuario.htm.

2 ČESKÝ STATISTICKÝ ÚŘAD (2018), Cizinci v $\check{C} R$ - včetně azylanti̊, [cit. 18.4.2019], recuperado de: https://www.czso.cz/csu/cizinci/cizinci-v-cr-vcetne-azylantu.

${ }^{3}$ A finales de diciembre de 2018, en la República Checa vivían aproximadamente 1670 españoles.

${ }^{4}$ La lengua $\mathrm{A}$ es la lengua materna del intérprete, la lengua $\mathrm{B}$ es la lengua que el intérprete domina perfectamente y a la que interpreta, y la lengua $\mathrm{C}$ es la lengua que el intérprete comprende perfectamente y de la que interpreta a su lengua A o B. Más información en: BAKER Mona, SALDANHA Gabriela (2009), Routledge encyclopedia of translation studies, London and New York, Routledge, p. 52.

${ }^{5}$ Entre otros, el Ministerio del Interior de la República Checa organiza cursos de integración destinados únicamente a los extranjeros provenientes de países externos a la Unión Europea, que son obligatorios a partir del año 2021.
} 
auditiva, cualquier desviación (ya sea fonética o léxica) de la variedad regional más común para el oyente (no solo para un intérprete) presenta una dificultad y puede afectar la capacidad de comprender y analizar el discurso. De igual modo, hace incrementar la carga de memoria de corta duración, deteriorando la capacidad de guardar la información por parte del intérprete. Partiendo del modelo de esfuerzos ${ }^{6}$ propuesto por Daniel GILE (2009: 160), se patentiza que un mayor esfuerzo para escuchar y analizar el texto original y para almacenar la información de manera temporal puede llevar al intérprete al borde de sus recursos cognitivos. Esto puede causar una saturación mental, que tendrá consecuencias negativas en la calidad de su interpretación.

En vista de lo anterior, resulta altamente recomendable que los estudiantes del programa Máster Interpretación checo-español estén en contacto con el mayor número posible de las variedades regionales del español, puesto que al ser expuestos a las diferentes variedades regionales de su lengua de trabajo, aprenden a utilizar las estrategias que mejorarán la calidad de su intepretación.

\section{El experimento}

Considerando los estudios mencionados, se formuló la siguiente pregunta de investigación: «¿Se pueden percibir diferencias en la calidad de la interpretación cuando se interpreta a los oradores de América Latina y a los de España?».

Para determinar el nivel del conocimiento de las variedades regionales del español entre los estudiantes de interpretación y su posible influencia en la calidad de la interpretación, se llevó a cabo un experimento con cinco estudiantes del programa Máster Interpretación checo-español en el Instituto de Traductología de la Facultad de Filosofía y Letras de la Universidad Carolina. ${ }^{7}$ En el momento de la realización del experimento, cuatro de los intérpretes estaban a punto de terminar su segundo semestre de interpretación simultánea, mientras que el quinto había asistido a un semestre de interpretación simultánea más.

Los estudiantes interpretaron un discurso no auténtico compuesto por cuatro partes. Cada parte del discurso fue pronunciada por una oradora de origen diferente: de Argentina, Perú, España y México. ${ }^{8}$ Tras interpretar simultáneamente el discurso, los estudiantes completaron un cuestionario de 25 preguntas.

La primera parte del discurso fue pronunciada por una oradora de Argentina, de Buenos Aires; la segunda, por una oradora de Perú, de la ciudad de Trujillo, situada

\footnotetext{
${ }^{6}$ Según el modelo de esfuerzos, la interpretación simultánea se compone de cuatro esfuerzos: esfuerzo de escucha y análisis, esfuerzo de memoria, esfuerzo de producción y esfuerzo de coordinación. ${ }^{7} \mathrm{El}$ experimento formó parte del TFM Vliv regionálních variant španélštiny na kvalitu simultánního tlumočeni do češtiny defendido por la autora del presente artículo en mayo 2019 en el Instituto de Traductología.

${ }^{8}$ En lo que se refiere a las variedades del español elegidas para el experimento, las variedades fueron seleccionadas, en parte, de acuerdo con la propia preferencia de la autora del experimento y, en parte, según la frecuencia con la cual los estudiantes pudieran estar en contacto con las variedades en cuestión. Por ejemplo, la variedad del español de México fue seleccionada principalmente por el motivo de que las personas provenientes de México constituyen el segundo grupo más numeroso de origen hispanohablante que reside en la República Checa, después de los españoles.
} 
en el noroeste; la tercera, por una oradora de España, de Álava; ${ }^{9}$ finalmente, la última, por una oradora de México, del Estado de Guanajuato.

En lo que se refiere al contenido del discurso original, se construyó un discurso de 10 minutos que fuera posible dividir en cuatro partes de igual duración y del mismo nivel de dificultad. El tema del discurso (Los efectos beneficiosos del café) fue elegido de conformidad con los planes curriculares del programa Máster Interpretación checo-español. ${ }^{10} \mathrm{El}$ discurso fue construido de tal manera que cada una de sus cuatro partes contuviera la misma cantidad de nombres propios, nombres de instituciones, enumeraciones y cifras, ya que dichos elementos pueden incrementar la carga de memoria de corta duración, lo que puede deteriorar la calidad de la interpretación y derivar en errores en la interpretación. Además, la velocidad de todas las partes del discurso era casi idéntica, en concreto, de entre 108 y 112 palabras por minuto. ${ }^{11}$ Acogiéndose a estos criterios, se garantizó que todas las partes del discurso original tuvieran el mismo nivel de dificultad.

Para asegurar la mayor naturalidad posible del discurso conforme a las respectivas variedades, se pidió a cada una de las oradoras que, antes de grabar su parte del discurso, leyera el texto y realizara todos los cambios oportunos para adecuarlo a su variedad del español, centrándose en particular en el léxico y la sintaxis.

A pesar de la reducida extensión del experimento, se ha creado un marco metodológico que puede ser modificado por las futuras investigaciones. No cabe duda de que el experimento descrito aquí podría repetirse utilizando un discurso diferente pronunciado por oradores provenientes de otras partes, no solo de América Latina, sino también de España.

\subsection{Errores de interpretación y su tipología}

El objetivo principal del experimento fue determinar si existe una diferencia entre los errores que cometen los futuros intérpretes al trabajar con los discursos pronunciados por hablantes del español latinoamericano, en comparación con el peninsular. Para ello, se elaboraron transcripciones de todas las interpretaciones y se analizó el número de omisiones, adiciones, sustituciones y de errores de presentación de cada intérprete. A tal efecto, fue utilizado el método de evaluación de la calidad de la interpretación propuesto por Henri BARIK en 1971 y elaborado por Andrzej KOPCZYŃSKI en 1980. Dentro de la tipología de errores, BARIK (1971: 200) identifica tres tipos de desviaciones de contenido y sentido respecto al discurso original: las omisiones, adiciones y sustituciones. Las omisiones son los elementos presentes en el discurso original que no están presentes en el discurso meta, es decir,

\footnotetext{
${ }^{9}$ La razón principal de dirigirse a esta oradora fue que la provincia de Álava, situada en el norte de España, se considera una de las zonas en las que se habla el español estándar, es decir, el español más cercano al que dicta la Real Academia Española. Más información sobre el español estándar y otras variedades del español en: KRÁlovÁ Jana (1998), Kapitoly ze zvukového rozboru španélštiny (na pozadí češtiny), Praha, Karolinum.

${ }^{10}$ Los primeros cursos de interpretación simultánea suelen centrarse en los temas más generales como, por ejemplo, la salud, el estilo de vida, las enfermedades, etc.

${ }^{11}$ Según la AIIC (Asociación Internacional de Intérpretes de Conferencias), la velocidad ideal de un discurso interpretado se sitúa entre las 100 y las 120 palabras por minuto. Más información en: PÖCHHACKER Franz (2004), Introducing interpreting studies, London, Routledge, p. 129.
} 
en la interpretación. ${ }^{12}$ Las adiciones son los elementos que no están presentes en el discurso original y que el intérprete añade al discurso meta. Se trata principalmente de calificadores y conectores. Las sustituciones son los segmentos de información del discurso meta que sustituyen los segmentos presentes en el discurso original y que alteran el sentido del mensaje. Esto significa que algunas sustituciones pueden ser leves, mientras que algunas pueden cambiar el sentido del mensaje original y alterar su idea principal.

KOPCZYŃSKI (1980: 82), aparte de elaborar la tipología de errores de interpretación, recomienda identificar un tipo de errores más: los errores de presentación. Se trata de falsos comienzos, pausas sonoras, titubeos y repeticiones, es decir, todos los errores que impiden que el intérprete transmita el mensaje con fluidez, lo cual puede ser percibido por los oyentes como falta de competencia. Partiendo de la tipología propuesta por BARIK, y teniendo en cuenta las sugerencias de KOPCZYŃSKI para modificarla, el presente artículo distingue entre omisiones tipo M1, M2 y M4, adiciones tipo A1 y A3, sustituciones tipo E1, E2 y E5, y errores de presentación señalados en las transcripciones con una $F$ (falsos comienzos), --- (pausas sonoras), Z (titubeos), y x (repeticiones). A diferencia de BARIK, que clasifica los errores de interpretación no solo a partir de la gravedad de la desviación, sino también según su causa, a efectos del presente estudio, se clasifican los errores basándose únicamente en el nivel de su gravedad. En consecuencia, no se distingue entre las omisiones tipo M2 y M3 causadas por falta de comprensión por parte del intérprete y falta de tiempo, respectivamente. Asimismo, por motivos de claridad, no se distingue entre las adiciones tipo A1 y A2, y entre las sustituciones tipo E1 y E3, y E2 y E4, puesto que la diferencia entre estos pares de errores consiste en la clase de palabras o la duración del elemento omitido, añadido o sustituido, y no en su gravedad.

Como ya se ha mencionado, a efectos del presente estudio, los errores solo se clasifican según su gravedad. Poniendo como ejemplo la categoría de las omisiones, una omisión tipo M1 aparte de no alterar la estructura gramatical de la oración, causa solamente poca pérdida de información. Una omisión tipo M2, en cambio, causa una mayor pérdida de información y, además, influye negativamente en la fluidez de expresión. Las omisiones tipo M4 causan una pérdida de información relevante $\mathrm{y}$, por lo tanto, pueden alterar la idea principal de la oración significativamente.

Para ilustrar la aplicación de la tipología de errores de interpretación, se introduce el siguiente ejemplo:

\footnotetext{
${ }^{12}$ Cabe mencionar que hay ocasiones en las que es deseable que el intérprete omita algunos elementos del discurso original para facilitar la comunicación entre el orador y el oyente. Al hablar de omisiones, el presente texto no se refiere a este tipo de omisiones estratégicas, sino a las omisiones de elementos relevantes, que pueden considerarse un error.
} 


\section{El discurso original:}

Somos muchos los que no podemos empezar el día sin una buena taza de café. De acuerdo con la Organización Internacional del Café, más del $30 \%$ de la población mundial consume a diario al menos una taza, con lo cual, el café logró hasta ahora posicionarse como la segunda bebida más consumida en el mundo; por detrás del agua, por supuesto.
La interpretación ${ }^{13}$ :

Je nás vice (E1), kteři nemůžeme začit den bez dobrého $(\mathrm{F})$ bez šálku dobré kávy. Vzpominám si (A3), že mezinárodní organizace kávy tvrdi, že (M1) $30 \%$ obyvatelstva planety si dává minimálně jeden šálek kávy denně. Takže káva dodnes --- obsadila druhé misto nejspotřbovávanějšiho nápoje. (M2)

Se puede observar que al comenzar, el intérprete cometió un error tipo E1, es decir, un error de semántica leve, seguido por un falso comienzo. Al principio de la segunda frase, el intérprete añadió en su interpretación una información que no estaba presente en el discurso original. Esta adición tipo A3 está seguida por una omisión leve tipo M1. La omisión causó cierta pérdida de información, pero no alteró el sentido del mensaje. Luego, el intérprete hizo una hesitación, o sea, una pausa sonora. Por último, el intérprete cometió una omisión tipo M2, que causó una pérdida de información más significativa.

\subsection{Los resultados del experimento}

En lo que se refiere a la pregunta de investigación planteada al inicio de este apartado, los resultados del experimento permiten deducir que diferentes variedades regionales del español tienen impacto en la calidad de la interpretación, en otras palabras, en el número de omisiones, adiciones, sustituciones y errores de presentación.

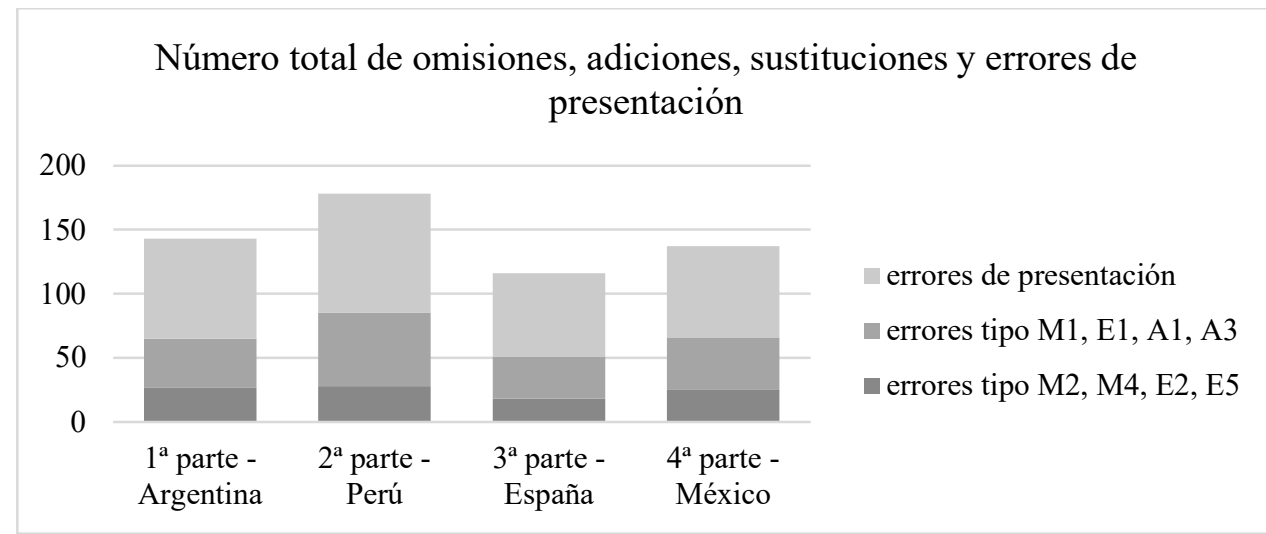

Figura 1. Número total de omisiones, adiciones, sustituciones y errores de presentación

En términos promedios, los resultados presentados en esta gráfica indican que al interpretar la segunda parte pronunciada por la oradora de Perú, los intérpretes cometieron el mayor número de errores. Por el contrario, al interpretar la tercera parte pronunciada por la oradora de España, los intérpretes cometieron el menor

\footnotetext{
${ }^{13}$ Traducción literal de la interpretación: Somos más (E1) los que no podemos empezar el día sin una buena (F) sin una buena taza de café. Recuerdo (A3) que la Organzación Internacional del Café dice que (M1) el $30 \%$ de la población mundial consume al menos una taza de café al día. Entonces, hasta ahora el café --- se ha posicionado como la segunda bebida más consumida. (M2)
} 
número de errores de todo tipo. A la hora de interpretar la primera parte presentada por la oradora de Argentina y la cuarta parte presentada por la oradora de México, los intérpretes cometieron prácticamente una cantidad idéntica de errores.

Como ya se ha comentado, tras interpretar simultáneamente el discurso, los intérpretes completaron cuestionarios de 25 preguntas. ${ }^{14}$ En relación con los cuestionarios utilizados durante el experimento, cabe destacar las respuestas de los intérpretes a la siguiente pregunta: «¿De qué país provienen las oradoras?». La siguiente figura muestra las respuestas individuales:

\begin{tabular}{|l|l|l|l|l|l|}
\hline & Intérprete 1 & Intérprete 2 & Intérprete 3 & Intérprete 4 & Intérprete 5 \\
\hline $\mathbf{1}^{\mathbf{a}}$ parte & Argentina & Argentina & Argentina & Argentina & Argentina \\
\hline $\mathbf{2}^{\mathbf{a}}$ parte & - & - & Salvador/Guatemala & Colombia & - \\
\hline $\mathbf{3}^{\mathrm{a}}$ parte & España & - & España & - & - \\
\hline $\mathbf{4}^{\mathbf{a}}$ parte & Venezuela & - & Méxice Chile & México & - \\
\hline
\end{tabular}

Figura 2. Respuestas de los participantes a la pregunta: «¿De qué país provienen las oradoras?»

Como puede observarse en la figura, todos los intérpretes determinaron correctamente de qué país provenía la oradora de Argentina. No obstante, ninguno de los intérpretes adivinó de qué país era la oradora de Perú. Dos de los intérpretes determinaron correctamente el país de origen de la oradora de España. Del mismo modo, dos de los intérpretes determinaron correctamente de qué país provenía la oradora de México, sin embargo, uno de ellos, al final, decidió tachar la respuesta correcta. $^{15}$

Relacionando los rendimientos de los intérpretes con sus respuestas a los cuestionarios, puede resultar interesante cómo los intérpretes respondieron a la siguiente pregunta: «Al interpretar del español al checo, ¿tiene preferencia respecto al lugar de origen del orador?». Se trató de una pregunta cerrada, los intérpretes debían elegir una de las siguientes respuestas disponibles: hablantes nativos de España, hablantes nativos de América Latina, no tengo preferencia. El único intérprete que no tiene ninguna preferencia al respecto, que es el Intérprete 2, cometió a lo largo del discurso menos errores de todo tipo que el resto de los intérpretes. Las respuestas a esta pregunta también muestran que dos participantes (el Intérprete 1 y el Intérprete 4) prefieren interpretar a los hablantes del español peninsular a interpretar a los hablantes del español latinoamericano, mientras que otros dos (el Intérprete 3 y el Intérprete 5), en cambio, prefieren interpretar a los oradores de origen latinoamericano. Es preciso destacar que el Intérprete 5 fue el segundo intérprete con menor número de errores y que el Intérprete 3 cometió en cada parte del discurso el mismo número de errores, es decir, a lo largo de todo el discurso, la calidad de su prestación no variaba.

\footnotetext{
${ }^{14}$ Los cuestionarios fueron diseñados siguiendo las recomendaciones de Sandra HALE y Jemina NAPIER en su libro Research methods in interpreting: a practical resource.

${ }^{15}$ En algunos casos, marcados en la figura con un guion, los intérpretes decidieron no responder.
} 
Otra característica importante se deriva de la comparación de los resultados del rendimiento de los intérpretes con sus respuestas a las siguientes preguntas del cuestionario: «¿Con qué frecuencia interpreta a las personas de España?», «¿Con qué frecuencia interpreta a las personas de América Latina?», «¿Con qué frecuencia escucha (TV, radio) a las personas de España?», «¿Con qué frecuencia escucha (TV, radio) a las personas de América Latina?». Todas estas preguntas eran cerradas y los intérpretes debían elegir una de las siguientes respuestas disponibles: muy a menudo, a menudo, a veces, casi nunca, nunca. Las respuestas están incluidas en la figura siguiente:

\begin{tabular}{|c|c|c|c|c|c|c|}
\hline & & $\begin{array}{l}\text { Intérprete } \\
1\end{array}$ & $\begin{array}{l}\text { Intérprete } \\
2\end{array}$ & $\begin{array}{l}\text { Intérprete } \\
\mathbf{3}\end{array}$ & $\begin{array}{l}\text { Intérprete } \\
4\end{array}$ & $\begin{array}{l}\text { Intérprete } \\
5\end{array}$ \\
\hline \multirow{2}{*}{$\begin{array}{l}\text { ¿Con qué } \\
\text { frecuencia } \\
\text { interpreta } \\
\text { a... }\end{array}$} & $\begin{array}{l}\text {...las } \\
\text { personas de } \\
\text { España? }\end{array}$ & $\begin{array}{l}\text { A } \\
\text { MENUDO }\end{array}$ & $\begin{array}{l}\text { A } \\
\text { MENUDO }\end{array}$ & $\begin{array}{l}\text { A } \\
\text { MENUDO }\end{array}$ & $\begin{array}{l}\text { A } \\
\text { MENUDO }\end{array}$ & A VECES \\
\hline & $\begin{array}{l}\text {...las } \\
\text { personas de } \\
\text { América } \\
\text { Latina? }\end{array}$ & $\begin{array}{l}\text { CASI } \\
\text { NUNCA }\end{array}$ & A VECES & A VECES & $\begin{array}{l}\text { CASI } \\
\text { NUNCA }\end{array}$ & $\begin{array}{l}\text { CASI } \\
\text { NUNCA }\end{array}$ \\
\hline \multirow{2}{*}{$\begin{array}{l}\text { ¿Con qué } \\
\text { frecuencia } \\
\text { escucha } \\
\text { (TV, radio) } \\
\text { a... }\end{array}$} & $\begin{array}{l}\text {...las } \\
\text { personas de } \\
\text { España? }\end{array}$ & A VECES & $\begin{array}{l}\text { A } \\
\text { MENUDO }\end{array}$ & $\begin{array}{l}\text { CASI } \\
\text { NUNCA }\end{array}$ & A VECES & A VECES \\
\hline & $\begin{array}{l}\text {...las } \\
\text { personas de } \\
\text { América } \\
\text { Latina? }\end{array}$ & NUNCA & $\begin{array}{l}\text { A } \\
\text { MENUDO }\end{array}$ & $\begin{array}{l}\text { CASI } \\
\text { NUNCA }\end{array}$ & $\begin{array}{l}\text { CASI } \\
\text { NUNCA }\end{array}$ & $\begin{array}{l}\text { A } \\
\text { MENUDO }\end{array}$ \\
\hline
\end{tabular}

Figura 3. Respuestas de los participantes sobre su contacto con las variedades del español

Cabe destacar que los Intérpretes 2 y 5 , que según sus respuestas están en contacto con el español latinoamericano a menudo, cometieron menos errores y produjeron una versión más correcta que los demás intérpretes. Por el contrario, el Intérprete 1, que no suele escuchar (en TV, radio) a los hispanohablantes de América Latina, cometió el mayor número de errores. No obstante, como se detalla más adelante, gracias a las nuevas tecnologías, los estudiantes pueden estar en contacto con los hispanohablantes de España y de América Latina en la misma medida.

A continuación, se presentan brevemente varios recursos en línea de que puede disponer cualquier estudiante de español como lengua extranjera, ya sea estudiante de interpretación o no, para mejorar su conocimiento de las variedades regionales de la lengua. Estos son algunos de los innumerables recursos en línea:

Speechpool es una base de datos que contiene un alto número de discursos pronunciados tanto por personas de España como de América Latina. Además, cada discurso incluye información sobre el país o incluso sobre la región del país de la

\footnotetext{
${ }^{16}$ Cabe mencionar que el cuestionario no diferenció entre la frecuencia con la cual los participantes interpretaban durante las clases en la universidad y la frecuencia con la cual interpretaban durante el autoaprendizaje, es decir, fuera de las clases. Durante las clases, los estudiantes del programa Máster Interpretación checo-español interpretan a los hablantes del español latinoamericano con regularidad, aunque en menor medida que a los hablantes del español peninsular.
} 
que proviene el orador. La plataforma también cuenta con los discursos pronunciados por hispanohablantes no nativos.

Speech Repository es una base de datos elaborada por la Unión Europea que comprende discursos en español presentados principalmente por hablantes del español peninsular. La plataforma también incluye un número de discursos presentados por oradores provenientes de Cuba.

TED en Español y TEDx son dos canales que contienen vídeos de charlas organizadas tanto en España como en América Latina. Los discursos tratan muchos temas distintos e interesantes.

Otros recursos útiles incluyen canales de Casa Rosada, Presidencia de la República - Colombia, Casa Presidencial Honduras, etc. Estos canales contienen declaraciones a la prensa, inauguraciones o visitas internacionales.

En la página web de YouTube está disponible una gran cantidad de programas de televisión en directo como, por ejemplo, Tv pública Argentina, Tv Venezuela o Tv MILENIO, para mencionar solamente unos pocos. Otros programas de televisión en directo están disponibles en las páginas web de las propias televisiones, entre otros, Radio y Televisión Española (RTVE).

\section{Conclusión}

Si bien es fundamental tomar en consideración que el número de participantes del presente estudio fue limitado, los resultados del experimento demuestran que los estudiantes de interpretación cometieron menos errores y produjeron una versión más correcta al interpretar el fragmento pronunciado por la oradora de origen español que al interpretar las partes del discurso pronunciadas por las oradoras de Argentina, Perú y México.

No obstante, a la hora de interpretar a las oradoras de América Latina, los estudiantes del programa Máster Interpretación checo-español que están acostumbrados a ver los programas de televisión de América Latina y los programas de España en la misma medida, encuentran menos dificultades que los demás intérpretes. Por lo tanto, se puede afirmar que una mayor exposición a las variedades regionales del español hace mejorar el rendimiento del intérprete, perfeccionando tanto su capacidad de comprender y analizar el discurso como la de guardar la información.

Por último y a pesar de las restricciones indicadas anteriormente, cabe mencionar que este trabajo pretende servir como estudio piloto destinado a ampliar la investigación sobre la evaluación de la calidad en interpretación simultánea.

\section{BIBLIOGRAFÍA}

BALOUNOVÁ Zuzana (2019), Vliv regionálních variant španělštiny na kvalitu simultánního tlumočení do češtiny, Praha, Univerzita Karlova, diplomová práce.

BALOUNOVÁ Zuzana (2019), Vliv regionálních variant španělštiny na kvalitu simultánního tlumočení do češtiny, en: MraČKOVÁ VAVROUŠOVÁ Petra, KLIMEŠ Lukáš (eds.), AUC Philologica 04/2019, p. 59-70. 
BAKER Mona, SALDANHA Gabriela (2009), Routledge encyclopedia of translation studies, London and New York, Routledge.

BARIK Henri (1971), A Description of Various Types of Omissions, Additions and Errors of Translation Encountered in Simultaneous Interpretation, en: Meta 16(4), p. 199-210.

ČESKÝ STATISTICKÝ ÚŘAD (2018), Cizinci v $\check{C} R$ - včetně azylantů, [cit. 18.4.2019], recuperado de: https://www.czso.cz/csu/cizinci/cizinci-v-cr-vcetne-azylantu.

GILE Daniel (2009), Basic concepts and models for interpreter and translator training, Amsterdam, John Benjamins.

HALE Sandra, NAPIER Jemina (2013), Research methods in interpreting: a practical resource, London, Bloomsbury.

INSTITUTO CERVANTES (2018), 577 millones de personas hablan español, el 7,6\% de la población mundial, [cit. 28.8.2019], recuperado de:

https://www.cervantes.es/sobre_instituto_cervantes/prensa/2018/noticias/np_p resentacion-anuario.htm.

KOPCZYŃSKI Andrzej (1980), Conference interpreting: some linguistic and communicative problems, Poznań, Universidad Adam Mickiewicz en Poznań.

KRÁLOVÁ Jana (1998), Kapitoly ze zvukového rozboru španělštiny (na pozadí češtiny), Praha, Karolinum.

PÖCHHACKER Franz (2004), Introducing interpreting studies, London, Routledge.

VAN ENGEN Kristin J., PEELLE Jonathan E. (2014), Listening effort and accented speech, en: Frontiers, [cit. 20.2.2019], recuperado de: https://www.frontiersin.org/articles/10.3389/fnhum.2014.00577/full

\section{PÁGINAS WeB}

CASA RosadA - RepÚBlica ARgentina, Casarosada, en: YouTube, recuperado de: https://www.youtube.com/user/casarosada.

MiLENIO, Milenio, en: Youtube, recuperado de: https://www.youtube.com/watch?v=1R6ElPjEttw.

PRESIDENCIA DE LA REPÚBLICA - COLOMBIA, Sigcolombia, en: YouTube, recuperado de: https://www.youtube.com/user/SIGCOLOMBIA/featured.

SPEECHPOOL, Speechpool, recuperado de: http://www.speechpool.net/es/.

SPEECH REPOSITORY 2.0, Speech Repository, recuperado de: https://webgate.ec.europa.eu/sr/.

TV PÚBliCA ARgentina, TV Pública Argentina, en: YouTube, recuperado de: https://www.youtube.com/watch? $=$ gDjglU2RukM.

TV VENEZUELA, TV Venezuela, en: YouTube, recuperado de: https://www.youtube.com/watch?v=iQ-VHIpaXRY. 